\title{
A study of the prevalence of group B streptococci in the third trimester of pregnancy
}

\author{
Vikas Tambe, Versha Shokeen*, Himadri Bal, Ajita Mishra
}

Department of Obstetrics and Gynecology, Dr. D. Y. Patil Medical College and Vidyapeeth, Pune, Maharashtra, India

Received: 14 June 2019

Accepted: 10 September 2019

\section{* Correspondence:}

Dr. Versha Shokeen,

E-mail: versha.shokeen@gmail.com

Copyright: (C) the author(s), publisher and licensee Medip Academy. This is an open-access article distributed under the terms of the Creative Commons Attribution Non-Commercial License, which permits unrestricted non-commercial use, distribution, and reproduction in any medium, provided the original work is properly cited.

\begin{abstract}
Background: Group B Streptococci (GBS) is an important cause of early onset neonatal sepsis and the maternal colonization of this organism is a key factor in the occurrence of GBS associated morbidity and mortality in the newborns. Timely recognition of its presence in the genital tract of a pregnant women and intrapartum antibiotic prophylaxis can significantly bring down the burden of the disease in neonates. A cross sectional study was conducted on antenatal women during 35-37weeks of gestation to evaluate the prevalence of Group B Streptococci in third trimester of pregnancy and explore the feasibility of including GBS screening in the routine antenatal investigation protocol.

Methods: 200 antenatal women satisfying the exclusion/inclusion criteria were recruited for the study. Vaginal and perianal swabs were collected using sterile swab sticks and inoculated using the specified media. Beta hemolysis and typical colonies were looked for under microscope. Positive cases were subjected to intrapartum antibiotic prophylaxis and the neonates were observed for 72 hours to look for any signs of sepsis.

Results: It was found that $2 \%$ of the women screened were positive for GBS .While none of the newborns of the 4 positive cases showed any signs of sepsis.

Conclusions: Prophylactic intrapartum prophylaxis against GBS has shown to decrease the chances of neonatal sepsis but more detailed and robust studies are required before incorporating routine screening in our antenatal care system.
\end{abstract}

Keywords: Antenatal screening, Early neonatal sepsis, Group B Streptococci, Intrapartum antibiotic prophylaxis, Neonatal sepsis, Vaginal and rectal colonisation

\section{INTRODUCTION}

Emerging as a major pathogen that affects newborns, Group B Streptococcus (GBS) has been a constituent of the normal microflora in our system. ${ }^{1}$ It may not demonstrate any clinical symptoms although the vagina and the peri-anal regions/rectum form its major reservoirs. However, in pregnant women and newborns, the colonization of these regions developed to be a risk factor for subsequent infection. ${ }^{2}$ Around $50-60 \%$ of infants have positive GBS cultures from skin and mucous membranes amongst those born to colonized mothers and $1-2 \%$ of these colonized newborns acquire invasive GBS infection. Maternal chemoprophylaxis at the time of delivery can mitigate the possibility of transfer from mother to newborn and, thus may diminish the number of infected cases and also the severity of the disease. ${ }^{3}$

Nevertheless, Indian studies among infants, show a domination of Gram negative bacterial sepsis which is conducive to infant mortality. On that account, there is a possibility of the role of GBS being underestimated or under 
reported. ${ }^{4}$ The rate of GBS colonization in the vagina and/or rectum amongst pregnant women depends upon a variety of ethnic groups, geographic areas and age. Globally, maternal colonization by GBS was contemplated to range from $4 \%$ to $40 \%$ in several studies. ${ }^{3}$

In a developing country like India, neonatal mortality is a major concern where sepsis neonatorum keeps neonatologists on their toes in NICUs nationwide. Although in the western countries, organisms in the newborns implicated as a cause of sepsis are different, it will be irrational to overlook the role of GBS as a causative agent of this potentially fatal entity. This is where the role of screening all pregnant women for GBS as a commensal in their lower genital tracts lies. Therefore, the present study aims to determine the prevalence of GBS in pregnant females by screening them in the third trimester of pregnancy.

\section{METHODS}

A cross sectional study was conducted in a tertiary care hospital from June 2016 to June 2018 to evaluate the prevalence of Group B Streptococci in third trimester of pregnancy after clearance from ethical committee was obtained.

\section{Inclusion criteria}

- All antenatal patients between 35-37 weeks of gestation.

\section{Exclusion criteria}

- Antenatal patients with florid vaginal infection and patients in labour.

200 antenatal patients attending the OPD and IPD were recruited for the study after taking an informed consent. Prior to data collection, all patients were explained about the purpose of the study and ensured strict confidentiality. A detailed history was elicited and routine antenatal examination was carried out. The patients were made to lie in dorsal position with adequate exposure of vulvo-vaginal and perineal area under good light. With due aseptic precautions, the labia were separated to visualise the lower vagina. Vaginal swabs and perianal swabs were taken with sterile swab sticks. Swabs were inoculated into Stuarts Transport media. In the laboratory, the swabs were transferred to Todd Hewitt broth media containing nalidixic acid $15 \mathrm{mcg} / \mathrm{ml}$ and gentamycin sulphate $8 \mathrm{mcg} / \mathrm{ml}$ which is a selective enrichment broth media for GBS. The same were incubated at $37^{\circ} \mathrm{C}$ overnight. The swabs were plated on $5 \%$ sheep blood agar and incubated at 37 degree $\mathrm{C}$ for 24 hours. Typical colony morphology was observed for Beta hemolysis. Colonies showing Beta hemolysis were subjected to Gram stain, Hippurate hydrolysis, Catalase test and CAMP test. Those found positive were subjected to intrapartum antibiotic prophylaxis with injection ampicillin $2 \mathrm{~g}$ IV followed by $1 \mathrm{~g}$ IV every 4 hours till delivery and those allergic to penicillin group were given Inj. clindamycin $900 \mathrm{mg}$ IV 8 hourly till delivery. The neonates were followed up for 72 hours for any signs of sepsis.

\section{RESULTS}

The present study was undertaken to evaluate the prevalence of Group B streptococci in third trimester of pregnancy. A total of 200 antenatal patients between 3537 weeks of gestation were included our study.

Table 1: Maternal age of study population.

\begin{tabular}{|ll|l|}
\hline $\begin{array}{l}\text { Age of patients } \\
\text { (years) }\end{array}$ & $\begin{array}{l}\text { Number of } \\
\text { cases }\end{array}$ & $\begin{array}{l}\text { Percentage } \\
(\%)\end{array}$ \\
\hline $18-25$ & 130 & 65 \\
\hline $26-30$ & 59 & 29.5 \\
\hline$>30$ & 11 & 5.5 \\
\hline
\end{tabular}

It was found that maximum number $(65 \%)$ were aged 18 25 years, $29.5 \%$ of the patients were aged $26-30$ years and only $5.5 \%$ were above 30 years (Table 1 ). Hence younger population was predominant.

Table 2: Parity status of study population.

\begin{tabular}{|lll|}
\hline Parity & Number of cases & Percentage (\%) \\
\hline Primigravida & 104 & 52 \\
\hline Multigravida & 96 & 48 \\
\hline
\end{tabular}

It was observed that most of women, that is, $52 \%$ were primigravida (Table 2). This finding was commensurate with predominant younger women in the study.

Table 3: Distribution of birth weight of neonates.

\begin{tabular}{|lll|}
\hline $\begin{array}{l}\text { Birth weight } \\
\text { (kgs) }\end{array}$ & Number of neonates & $\begin{array}{l}\text { Percentage } \\
(\%)\end{array}$ \\
\hline$<2$ & 16 & 8 \\
\hline $2-2.49$ & 40 & 20 \\
\hline $2.5-3$ & 96 & 48 \\
\hline$>3$ & 48 & 24 \\
\hline
\end{tabular}

Most neonates (48\%) were appropriate for gestational age weighing 2.5 to $3 \mathrm{kgs} .24 \%$ of the neonates weighed more than $3 \mathrm{kgs}$. The remaining $28 \%$ of the neonates weighed between $2.49 \mathrm{kgs}$ to less than $2 \mathrm{~kg}(<2 \mathrm{~kg}-8 \%$ and 2 to $2.49 \mathrm{~kg}-20 \%$ ) (Table 3).

Table 4: Type of delivery.

\begin{tabular}{|lll|}
\hline Type of delivery & $\begin{array}{l}\text { Number } \\
\text { of delivery }\end{array}$ & Percentage $(\%)$ \\
\hline Vaginal & 145 & 72.5 \\
\hline Caesarean & 55 & 27.5 \\
\hline
\end{tabular}

Most of the mothers delivered vaginally. $72.5 \%$ the population under surveillance were full term normal 
delivery (FTND) while $27.5 \%$ were lower segment caesarean sections (Table 4).

Table 5: Prevalence of group B streptococci.

\begin{tabular}{|llll|}
$\begin{array}{l}\text { Swab } \\
\text { report }\end{array}$ & $\begin{array}{l}\text { Positive } \\
\text { for GBS }\end{array}$ & $\begin{array}{l}\text { Negative for } \\
\text { GBS }\end{array}$ & Total \\
\hline Number & 4 & 196 & 200 \\
\hline Percentage & 2 & 98 & 100 \\
\hline
\end{tabular}

GBS: Group B Streptococci.

Out of the 200 antenatal patients screened using vaginal and perianal swabs, only 4 participants i.e. $2.0 \%$ were found to be positive for Group B Streptococci (Table 5).

Table 6: Neonatal sepsis.

\begin{tabular}{|llll|}
\hline Neonatal sepsis & Present & Absent & Total \\
\hline Present & 0 & 0 & 0 \\
\hline Absent & 0 & 200 & 200 \\
\hline Total & $\mathbf{0}$ & $\mathbf{2 0 0}$ & $\mathbf{2 0 0}$ \\
\hline
\end{tabular}

Those found positive were subjected to intrapartum antibiotic prophylaxis and their neonates were observed for any signs of sepsis like fever, lethargy, irritability, refusal to feeds, seizures etc. None of the neonates of the GBS positive mothers showed any feature of sepsis.

The prevalence of GBS was only $2 \%$ amongst our study population that comprised of 200 women in their third trimester of pregnancy. Intra partum antibiotic prophylaxis as per the CDC guidelines proved effective in preventing neonatal sepsis in the neonates of the GBS positive mothers thus helping in reducing burden of the possible fatal disease.

\section{DISCUSSION}

In many parts of the world, Group B Streptococcus (GBS) is taken into consideration as an important cause of maternal and neonatal mortality and morbidity specially in Western countries in early 1970s. ${ }^{5}$ During labor, there has been an extensive adoption of antibiotic prophylaxis in numerous developed nations by 1990 s. $^{6}$ The perception that maternal colonization with the organism is pivotal to the manifestation of GBS-related neonatal sepsis formed the basis for the preventive strategies. Intra partum use of prophylactic antibiotics has unquestionably lead to a subsequent reduction in the rate of neonatal GBS disease. Nonetheless, in India, the role of GBS in neonatal morbidity and mortality is largely unrecognised and also underestimated. ${ }^{7}$

In the present study, 200 antenatal women were recruited for the screening of GBS colonization, $52 \%$ of women were observed to be primiparous and $48 \%$ were multiparous. It was also observed that most of the women had full term normal deliveries and majority of the newborns had satisfactory birth weight and birth outcome.
GBS was isolated in 4 women i.e. $2 \%$ of the patients. These women were followed up and were subjected to prophylactic antibiotics during labour which included ampicillin and those allergic to ampicillin were administered clindamycin as a part of prophylaxis against GBS. The neonates of GBS positive mothers were followed up and it was found that none developed any signs of neonatal sepsis.

A prevalence of $2.0 \%$ that was found was in accordance with other studies conducted in India, such as those by Vijayan et al, Uday et al and Santhanam et al, which have found a prevalence ranging from $2.3 \%$ to $2.6 \% .^{8-10}$

Overall the results of GBS maternal colonization varies from place to place and this may be due to various factors. Genetic, socio-economic and ethnic factors might play a pivotal role in the discrepancy of the rates of infection with GBS. Another possible reason for such variability include variation in clinical practices of sample collection as it has been observed that better sampling has led to isolation of more cases by studies in the past. Secondly, our country lacks set guidelines for the screening of mothers for GBS colonization and no special efforts are made to isolate GBS as the cause of sepsis in the neonates. Thirdly, inspite of major improvements in the health care sector over the decades, there is still a substantial number of home deliveries in our country. Most women are treated at rural centers during their antenatal period with no visits to tertiary care centers where facilities to screen them for GBS colonization can be made possible. Researches in the past have not recognized GBS as a major cause of health care issue in our country but what should not be ignored is that there is a possibility that the real problem has still remained unrecognized due to the lack of an organized screening program and what we have been looking at so far may be just the tip of an iceberg. This is where the question arises that have we been missing out on the actual threat that GBS poses in our country and do we need to re-consider the strategies that have been followed so far. To find answer to this question, there may be a need for more robust studies to decipher the real burden of the disease. This will help in deciding whether there is a case for implementing screening of all pregnant women for GBS colonization as a part of the routine antenatal care in our country.

\section{CONCLUSION}

Studies all over the country have shown a prevalence ranging from $2-7.6 \%$ but with a varying sample size.

Most of the available information on epidemiology of GBS in pregnancy is based on single observation of patients and longitudinal data are limited. Pilot studies, as the present one may provide an impetus for further research on GBS carriage, risk factors and their association with neonatal infection. 
GBS related neonatal sepsis in developed countries has led to guidelines by bodies like CDC and ACOG for GBS screening in pregnancy. Further robust studies under standardised conditions with larger sample sizes are required for evaluating the real threat of GBS in pregnancy in India and thereafter decide future course of action.

Funding: No funding sources Conflict of interest: None declared

Ethical approval: The study was approved by the Institutional Ethics Committee

\section{REFERENCES}

1. Schuchat A, Whitney C, Zangwill K. Prevention of perinatal group B streptococcal disease: a public health perspective. Centers for Disease Control and Prevention. MMWR Recomm Rep. 1996;45(RR7):1-24.

2. Gil EG, Rodríguez MC, Bartolomé R, Berjano B, Cabero L, Andreu A. Evaluation of the Granada agar plate for detection of vaginal and rectal group B streptococci in pregnant women. J Clin Microbiol. 1999;37(8):2648-51.

3. Bevilacqua G. Prevention of perinatal infection caused by group B beta-hemolytic streptococcus. Acta Biomed Ateneo Parmense. 1999;70(5-6):87-94.

4. Hoogkamp-Korstanje JA, Gerards LJ, Cats BP. Maternal carriage and neonatal acquisition of group B streptococci. J Infect Dis. 1982;145(6):800-3.
5. Gilbert R. Prenatal screening for group B streptococcal infection: Gaps in the evidence. Int $\mathbf{J}$ Epidemiol. 2004;33:2-8.

6. Shet A, Ferrieri P. Neonatal and maternal group B streptococcal infections: a comprehensive review. Indian J Med Res. 2004;120:141-50.

7. Baker CJ, Clark DJ, Barrett FF. Selective broth medium for isolation of group B streptococci. Appl Microbiol. 1973;26(6):884-5.

8. Uday R, Shetty MB, Dev G, Shivananjaiah C. Prevalence of group B streptococcus (GBS) in third trimester - A prospective cross sectional study. Int $\mathbf{J}$ Med Health Res. 2015;1(3):8-11.

9. Santhanam S, Jose R, Sahni RD, Thomas N, Beck MM. Prevalence of group B Streptococcal colonization among pregnant women and neonates in a tertiary hospital in India. J Turk Ger Gynecol Assoc. 2017;18(4):181-4.

10. Hajare V, Madhavi LH, Singh HK. Antibiogram of Group B Streptococci Isolated from the vagina of pregnant women in third trimester of pregnancy. People's J Sci. 2012;5(2):52-7.

Cite this article as: Tambe V, Shokeen V, Bal H, Mishra A. A study of the prevalence of group B streptococci in the third trimester of pregnancy. Int $\mathrm{J}$ Reprod Contracept Obstet Gynecol 2019;8:3911-4. 